EPJ manuscript No.

(will be inserted by the editor)

\title{
Power laws in elementary and heavy-ion collisions
}

\section{- A story of fluctuations and nonextensivity? -}

\author{
Grzegorz Wilk ${ }^{1}$ a and Zbigniew Włodarczyk ${ }^{2}$ b \\ 1 The Andrzej Soltan Institute for Nuclear Studies, Hoża 69; 00-689 Warsaw, Poland \\ 2 Institute of Physics, Jan Kochanowski University, Świętokrzyska 15, 25-406 Kielce, Poland \\ Received: date / Revised version: date
}

\begin{abstract}
We review from the point of view of nonextensive statistics the ubiquitous presence in elementary and heavy-ion collisions of power-law distributions. Special emphasis is placed on the conjecture that this is just a reflection of some intrinsic fluctuations existing in the hadronic systems considered. These systems summarily described by a single parameter $q$ playing the role of a nonextensivity measure in the nonextensive statistical models based on Tsallis entropy.
\end{abstract}

PACS. 89.75.-k complex systems - 24.60.-k statistical theory and fluctuations - 25.75.Dw particle production (relativistic collisions) - 25.75.-q relativistic heavy ions collision

\section{Introduction}

In many domains of physics, especially in elementary and heavy-ion collisions, for decades the prevailing understanding was that exponential shape of most of the observed spectra of produced secondaries suggests their statistical (or even thermodynamical) origin. Therefore, when looking for spectra of transverse momenta it is commonly assumed that the observed inverse mean transverse momenta (characterizing the widths of such exponential spec' tra) play the role of a temperature of the hadronizing system [1. This assumption allows us to use, in what follows, the whole machinery of statistical models. This is specially important in the case of high energy collisions of heavy ions because it allows us to use the tools of statistical physics to investigate the possibility of the formation in such collisions of a new state of matter, the so called Quark Gluon Plasma (QGP) 2] (in this case one is interested in details of hadron $\Longleftrightarrow Q G P$ phase transition, which can only be investigated in this way).

However, one should always keep in mind another possibility, namely that such behavior can just be due to the fact that, when presenting our data, out of many particles produced (tens or thousands at present, say $N$ to be specific) we select only one to make the corresponding plots. Single particle distributions averaged over many events are what is usually published. But this means that the remaining $(N-1)$ particles will act as a kind of heath bath. Assuming that this heath bath is homogenous and large it is natural to expect that its action can be described by a single parameter, which we then call temperature, $T$, and

\footnotetext{
a e-mail: wilk@fuw.edu.pl

b e-mail: wlod@pu.kielce.pl
}

identify with the temperature encountered in statistical models [3]. What one apparently observes then is just the usual Boltzmann-Gibbs (BG) statistics at work.

The above reasoning assumes that any dynamics of the set of remaining $(N-1)$ particles is mostly averaged and what results looks very much like a state of hadronic matter remaining in thermal equilibrium characterized by a temperature $T$. Some effects, however, can survive this equilibration process and can show up as apparent departures from the assumed thermal equilibrium. This is usually regarded as a departure from BG statistics and, finally, considered as a kind of failure of the simple statistical approach. Such observations are therefore a subject of separate investigations in which many different (dynamical) ideas compete (like, for example, the production of resonances and the flow of matter, to name only two) 4 . However, because there is only a limited amount of available data, usually one cannot decide which of the proposed dynamical remedies is right or, if we agree that they should all be present, in what proportion they show up 1

On the other hand, one can argue that, perhaps, it is the form of statistical model used which should be modified in such a way as to account (at least to some extent) for detected irregularities (i.e., for departures from the BG approach). Therefore, instead of inventing and investigating different dynamical assumptions, one can instead

\footnotetext{
1 This is connected with the important problem of how much information given measurements are providing us with. This is, so far, only sporadically discussed in the domain of high energy multiparticle production processes using information theory approach in which the entropies mentioned here are regarded as measures of information [5,6. This subject is, however, beyond the scope of our review.
} 
Grzegorz Wilk, Zbigniew Włodarczyk: Power laws in elementary and heavy-ion collisions

investigate the possibility of replacing the usual statistical model based on BG entropy, $S_{B G}$, by its modified version based on some other form of entropy discussed in the literature 7]. Such models are widely known nowadays from other branches of physics and are used whenever a physical system under investigation shows memory effects of any kind, experiences long range correlations (i.e., is in a sense "small" because its size is comparable with the range of forces acting in it), experiences some intrinsic fluctuations, or the phase space in which it operates is limited or has fractal structure. In this review we shall discuss the application of such a model taken in the form proposed by Tsallis, i.e., in the form based on the so called Tsallis entropy, $S_{T}=S_{q}[8$ :

$$
S_{q}=\frac{\left(1-\sum_{i} p_{i}^{q}\right)}{q-1} \stackrel{q \rightarrow 1}{\Longrightarrow} \quad S_{B G}=-\sum_{i} p_{i} \ln p_{i}
$$

Notice that $S_{B G}=S_{q=1}$. The $S_{q}$ is nonextensive because for any two independent systems $A$ and $B$ (in the usual sense, i.e., for which $\left.p_{i j}(A+B)=p_{i}(A) p_{j}(B)\right)$, one observes that

$$
S_{q}(A+B)=S_{q}(A)+S_{q}(B)+(1-q) S_{q}(A) S_{q}(B),
$$

In this sense the entropic index $q$ is a measure of the nonextensivity in the system without, however, directly showing up its cause. This must be provided from elsewhere.

To shed more light on the physics involved here, let us come back to the previous reasoning with some effective thermal bath being formed by the $(N-1)$ particles remaining after selection of the one used for making final histograms. Notice that, in high energy multiparticle production reactions observed in elementary and heavy-ion collisions we are interested in here, such thermal baths usually (i.e., after more detailed scrutiny) do not satisfy conditions allowing us to introduce the notion of thermal equilibrium in the BG sense: they are always finite and can be hardly considered as being homogenous (in fact, in many cases they occupy only a fraction of the allowed phase space [9] or even have a fractal-like structure [10] and it is known that usually the hadronizing system under consideration experiences long range correlations). This means therefore that such a heath bath cannot be described by a single parameter $T$. The simplest thing to do seems to be to allow for some fluctuations of the parameter $T$ and to replace it by its mean value $T \rightarrow T_{0}=\langle T\rangle$ and by one more parameter describing its fluctuations, using, for example, the (normalized) variance [11,12]:

$$
\omega=\frac{\left\langle\left(\frac{1}{T}\right)^{2}\right\rangle-\left\langle\frac{1}{T}\right\rangle^{2}}{\left\langle\frac{1}{T}\right\rangle^{2}} .
$$

In this approach

$$
q=1+\omega
$$

It can be next shown that such a heath bath leads in a natural way to the following $q$-exponential distribution (called also Tsallis distributions)2:

$$
\exp \left(-\frac{X}{\lambda}\right) \Rightarrow \exp _{q}\left(-\frac{X}{\lambda}\right)=\left[1-(1-q) \frac{X}{\lambda}\right]^{\frac{1}{1-q}}
$$

This is the power law we were searching for in different reactions [6, 13, 15, 16, 17, 18, 19,20,21,22, the results of which will be reviewed her 3 .

The physical picture presented above can be made more formal by saying that one replaces here the notion of strict local thermal equilibrium, customarily assumed in all applications of statistical models, by the notion of some kind of stationary state, which is being formed in the collision and which already includes some interactions. This concept can be introduced in different ways. For example, in [29] it was a random distortion of energy and momentum conservation caused by the surrounding system which resulted in the emergence of some nonextensive equilibrium. In 30,31 the two-body energy composition in transport theory formulation of the collision process is replaced by a generalized energy sum, $h\left(E_{1}, E_{2}\right)$, which is assumed to be associative but which is not necessarily simple addition and contains contributions stemming from pair interaction (in the simplest case). It turns out that under quite general assumptions about the function $h$, a division of the total energy among free particles is possible. Different forms of the function $h$ then lead to different forms of entropy formula, among which one encounters the known Tsallis form. The origin of this kind of thinking can be traced back to the analysis of the $q$-Hagedorn model proposed some time ago in 32 .

We close this section with a historical note. The recognition that some, apparently unexpected power law distributions can be due to fluctuations came to us from the observation in cosmic ray physics 33 . that there exists a long flying component (LFC) phenomenon in the propagation of the initial flux of incoming nucleons. For example, instead of the normally expected exponential fall off of the depth distribution of the starting points of cascades, $z$,

$$
\frac{d N(z)}{d z}=\text { const } \cdot \exp (-z / \lambda)
$$

one rather observes an $\exp _{q}(-z / \lambda)$ distribution, i.e., Tsallis like power law behavior given by Eq. (5) with $z$ replacing $X$ (here $\lambda \sim 1 / \sigma$ is the mean free path describing propagation of the incoming flux in the atmosphere with $\sigma$ being the relevant cross section). On the other hand, some time ago we have shown in [34] that this effect

\footnotetext{
${ }^{2}$ Except for 12 discussion concerning the meaning of the parameter $q$ was limited to the case of $q>1$ only. As already mentioned in 12, the case of $q<1$ seems not so much connected with any genuine fluctuations but rather, in some way, with limitations of the allowed phase space [13. It is worth mention that the idea of possible fluctuations of otherwise intensive quantities has already been formalized by introducing a new concept of so called superstatistics [14].

3 It should be noticed that there are also some other investigations on this subject $23,24,25,26,27,28$, with rather similar conclusions but which we will not discuss here.
} 
can be explained by assuming that hadronic cross sections should be regarded as fluctuating quantities with widths (defined as normalized dispersion), $\omega_{\sigma}=\left\langle\sigma^{2}\right\rangle /\langle\sigma\rangle^{2}-1$ (and growing logaritmically with energy). We were at that time prompted by the fact that such an idea was widely investigated in the usual hadronic collisions [35.4. It was then quite natural to connect the parameter $q$ with fluctuations. This done in 11, 12, as mentioned above.

We shall review our results in this field in the next Section. Section 3 contains some new recent developments in this field. The final Section contains our conclusions and a summary.

\section{Review of fluctuations in multiparticle production processes}

High energy collisions result in a multitude of particles of different kinds being produced. Most are just mesons of all kinds (overwhelmingly pions). For those who are looking for some new and/or rare phenomena they form unwanted background which must somehow be substracted, for others they are a subject of thorough investigations allowing us to look inside the very early stages of the collision process as well as at the hadronization stage of the matter produced (proceeding probably via the formation of the QGP, for example). In both cases a simple and trustworthy representation of data is very important, this justifies our investigations in this field to be reported here.

\subsection{Generalized heat bath - fluctuations of temperature}

We first recall the physical picture behind the generalized heat bath introduced in Section 1 which we have proposed in 11, 12. Our reasoning was as follows. Suppose we have a thermodynamic system, in a small (mentally separated) parts of which the temperature can take different values, i.e., in the whole system it fluctuates with $\Delta T \sim T$. Let $\xi(t)$ describes stochastic changes of temperature in time. If the mean temperature of the system temperature is $\langle T\rangle=$ $T_{0}$ then, as a result of fluctuations in some small selected region, the actual temperature $T^{\prime}$ equals

$$
T^{\prime}=T_{0}-b \xi(t) T
$$

where the constant $b$ is defined by the actual definition of the stochastic process under consideration, i.e., by $\xi(t)$, which is assumed to satisfy the condition that

$$
\langle\xi(t)\rangle=0
$$

and which correlator, $\langle\xi(t) \xi(t+\Delta t)\rangle$, for sufficiently fast changes is equal to

$$
\langle\xi(t) \xi(t+\Delta t)\rangle=2 D \delta(\Delta t) .
$$

\footnotetext{
${ }^{4}$ It is interesting to notice that this idea has been revitalized very recently in 36] and connected with the fluctuations in the gluonic content of hadrons.
}

The inevitable exchange of heat between any selected region of the system and the rest leads to equilibration of the temperature in the whole system. The corresponding process of heat conductance is described by the following equation 37,

$$
c_{p} \rho \frac{\partial T}{\partial t}-a\left(T^{\prime}-T\right)=0,
$$

where $c_{p}, \rho$ and $a$ are, respectively, the specific heat under constant pressure, density and the coefficient of external conductance. Using $T^{\prime}$ as defined in (7) we finally get the linear differential equation for the temperature $T$ with $\tau=$ $b=\frac{c_{p} \rho}{a}$ :

$$
\frac{\partial T}{\partial t}+\left[\frac{1}{\tau}+\xi(t)\right] T=\frac{1}{\tau} T_{0} .
$$

It can be now shown that this equation leads to the Langevin equation with multiplicative noise term resulting in fluctuations of the temperature $T$ given in the form of a gamma function [1]

$$
f(T)=\frac{1}{\Gamma(\alpha)} \mu\left(\frac{\mu}{T}\right)^{\alpha-1} \exp \left(-\frac{\mu}{T}\right)
$$

and characterized by the parameters $\mu$ and $\alpha$,

$$
\mu=\frac{\phi}{D} \quad \text { and } \quad \alpha=\frac{1}{q-1}=\frac{1}{\tau D} .
$$

This is to be compared with Eq. (3) in which now $\omega=\tau D$. Function $f(T)$ as given by Eq. (12) is the distribution that should be used to smear the parameter $T$ in the usual exponential distribution of the BG statistical model and which results in the Tsallis distribution, Eq. (5). To summarize: a small addition of the multiplicative noise described by a damping constant in the Langevin equation results in a stationary distribution of particle momenta, which develops a power-law tail at high values 5 .

\subsection{Transverse and longitudinal dynamics}

To begin our presentation we first set the stage. The characteristic pattern of the multiparticle production processes is that most of the secondaries are produced with small transverse momenta $p_{T}$ (mostly below $1 \mathrm{GeV}$ ) and are therefore concentrated in the longitudinal phase space given by the longitudinal momenta $p_{L}$ (which is described in terms of the rapidity $y=\frac{1}{2} \ln \frac{E+p_{L}}{E-p_{L}}$, where $E=\sqrt{m_{T}^{2}+p_{L}^{2}}$ with $m_{T}=\sqrt{m^{2}+p_{T}^{2}}$ being the so called transverse mass ( $m$ is mass of the particle); in other notation $E=m_{T} \cosh y$ and $\left.p_{L}=m_{T} \sinh y\right)$. The terms transverse and longitudinal are defined with respect to the direction of the colliding particles. Data are presented as distributions either

\footnotetext{
5 Actually, as shown in 14 when discussing superstatistics, there is a whole class of functions leading from $\exp (X)$ to $\exp _{q}(X)$. But only this has a simple physical interpretation as presented here. More general version of Langevin equation containing also additive noise have been considered in 38.
} 
in $p_{T}$ or in $y$. In both cases they show exponential behavior either in $p_{T}$ or in the energy $E=\left\langle m_{T}\right\rangle \cosh y$ (with $\left.\left\langle m_{T}\right\rangle=\sqrt{m^{2}+\left\langle p_{T}\right\rangle^{2}}\right)$ :

$$
\frac{d N}{d p_{T}}=C_{p_{T}} p_{T} \exp \left(-\frac{p_{T}}{T}\right) ; \quad \frac{d N}{d y}=C_{y} \exp \left(-\frac{E}{T}\right) .
$$

One observes dramatic differences in both distributions reflected by the differences in the values of the parameter $T$, which is of the order of one hundred $\mathrm{MeV}$ in $p_{T}$ space (where $T=T_{p_{T}}$ and is universal, i.e., essentially energy independent) and tens of GeV (depending on the energy of collision) in $p_{L}$ (or $y$ ) space (where $T=T_{p_{L}}$ and depends on energy). This means that the two distributions reflect different physics: those in $p_{T}$ space are believed to be essentially "thermal-like" and subject to a thermodynamic interpretation whereas, those in $p_{L}$ space are sensitive to the available energy and to the multiplicity of produced secondaries. Because of this their fluctuation patterns will be different, i.e., when described by Tsallis power-like form Eq. (5) the corresponding parameters $\left(q_{T}-1\right)$ and $\left(q_{L}-1\right)$ will differ dramatically. Also the physical meaning of these parameters will be different reflecting different sources of fluctuations.

\subsection{Longitudinal phase space}

We start with the longitudinal distribution in rapidity (averaged over $\left.p_{T}\right)$. In Fig. 1 one observes that $q<1$ is effectively cutting off the allowed longitudinal phase space (here defined by the initial available energy $M=100 \mathrm{GeV}$ and assumed constant transverse mass $m_{T}=0.44 \mathrm{GeV}$ and weakly depending on the assumed multiplicity of the produced particles $N$ ). Actually, from Eq. (5) it is obvious that only such combinations of $q$ and $X$ and $\lambda$ are allowed for which $[1-(1-q) X / \lambda]>0$. In [13, when fitting longitudinal distributions without restricting the available energy by introducing the so called inelasticity coefficient $K<1$, the only role of $q$, which was found to be $q<1$ there, was to limit the amount of energy used (showing the necessity of introducing inelasticity when considering multiparticle production processes, cf., 39 for review on this subject). For $q>1$ one observes a visible enhancement of distribution tails.

Physica A 344, 568 (2004)

The examples of fits to the actually observed single particle distributions in rapidity are shown in Figs. 2 and 4. In the left panel of Fig. 22 (see [19] for details) results for $p p$ and $p \bar{p}$ collisions at energies varying between $\sqrt{s}=20$ $\mathrm{GeV}$ to $1800 \mathrm{GeV}$ are displayed. From each listed energy of collision, $E_{c m}=\sqrt{s}$, only a fraction $K_{q}$ has been used for the production of secondaries (according to 39]). The other input was the mean multiplicity of charged secondaries produced in nonsingle diffractive reactions at given energy: $\bar{n}_{c h}=-7.0+7.2 s^{0.127}[1]$ (corresponding to the total number of produced particles, $\left.N=\frac{3}{2} \bar{n}_{c h}\right)$. The allowed phase space is one dimensional with only a small energy dependence of the mean transverse momentum allowed, $\left\langle p_{T}\right\rangle=0.3+0.044 \ln (\sqrt{s} / 20)$ [1] (all secondaries will be assumed to be pions of mass $\mu=0.14 \mathrm{GeV}$ ).

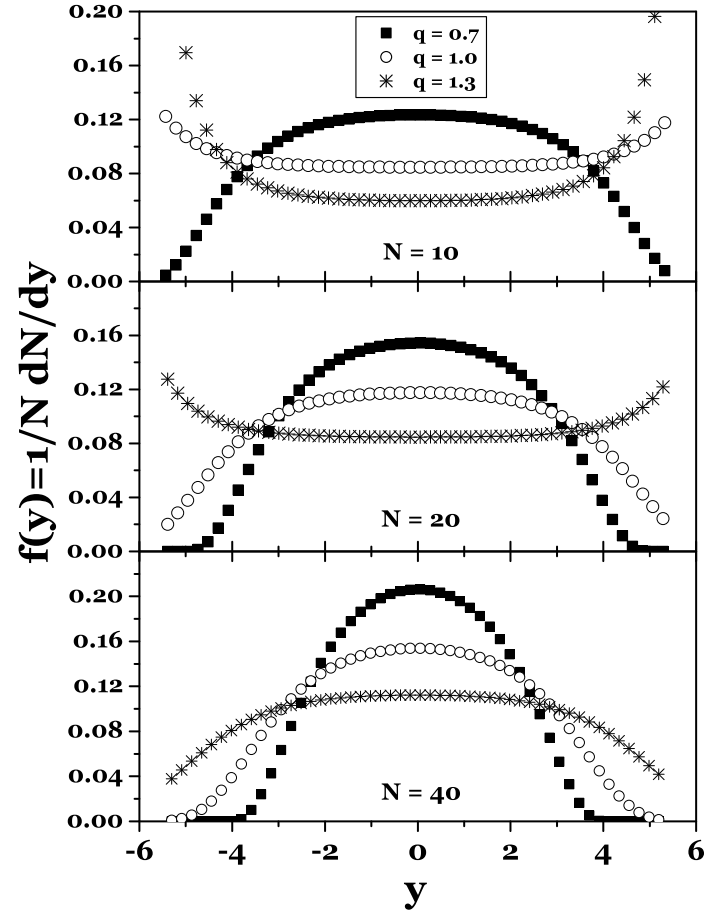

Fig. 1. Examples of the most probable rapidity distributions as given by Eq. (14) for hadronizing mass $M=100 \mathrm{GeV}$ decaying into $\mathrm{N}$ secondaries of (transverse) mass $m_{T}=0.4 \mathrm{GeV}$ each for different values of parameter $q=Q_{L}$ (reproduced by permission of Springer-Verlag from [18]).

As discussed in detail in 19, one gets in this case not only the parameter $q$ but also the true inelasticity, $K$, of the reaction (in fact, even, for the first time, its distribution, $\chi(K))$. Let us, however, concentrate on the parameter $q$, which bears information on fluctuations. It turns out to be energy dependent as presented in Fig. 3. Surprisingly enough it turned out that the same behavior is observed for the inverse of $k$ characterizing the so called Negative Binomial distribution (NB) [1 of the multiplicity of observed secondaries, which depends on two parameters: the mean multiplicity $\left\langle n_{c h}\right\rangle$ and the parameter $k(k \geq 1)$ affecting its width $(\sigma) n_{c h}$ is dispersion),

$$
\frac{1}{k}=\frac{\sigma^{2}\left(n_{c h}\right)}{\left\langle n_{c h}\right\rangle^{2}}-\frac{1}{\left\langle n_{c h}\right\rangle} .
$$

For $k \rightarrow 1 \mathrm{NB}$ approaches a geometrical distribution whereas for $k^{-1} \rightarrow 0$ it approaches a Poissonian distribution. In general it is found [1] that $\frac{1}{k}=-0.104+0.058 \cdot \ln \sqrt{s}$, which fits the obtained values very nicely, cf. Fig. 3 .

To fully understand the possible physical meaning of the parameter $q\left(=q_{L}\right)$ in this case let us remind ourselves that, in general, the nonextensivity parameter $q$ summarizes the action of several factors, each of which leads to a deviation from the simple form of the extensive BG statistics, as was mentioned before, out of which we are interested most in the possible intrinsic fluctuations existing in the hadronizing system [11. Notice that in our fits we have not explicitly accounted for the fact that each event has its own multiplicity, $N$, but we have 

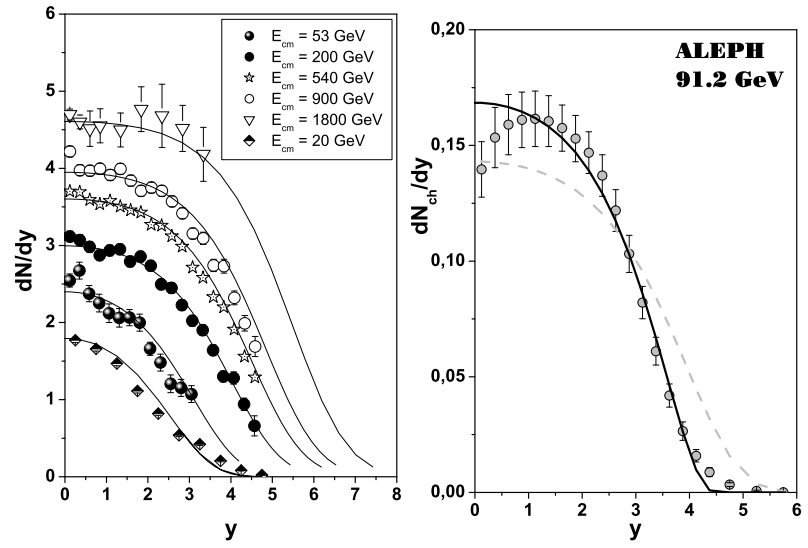

Fig. 2. Examples of applying the nonextensive approach to longitudinal distributions. Left panel: fit to rapidity spectra for charged pions produced in $p p$ and $\bar{p} p$ collisions at different energies [40. Right panel: rapidity spectra measured in $e^{+} e^{-}$annihilations at $91.2 \mathrm{GeV}$ [1] (dotted line is for $K_{q}=1$ and $q=1$ whereas the full line is our fit with $K_{q}=1$ and $q=0.6$ ). (Reprinted from Physica A344, F.S. Navarra, O.V. Utyuzh, G. Wilk and Z. Włodarczyk, "Information theory in high-energy physics (extensive and nonextensive approach)", 568, Copyright (2004), with permission from Elsevier; http://www.elsevier.com ).

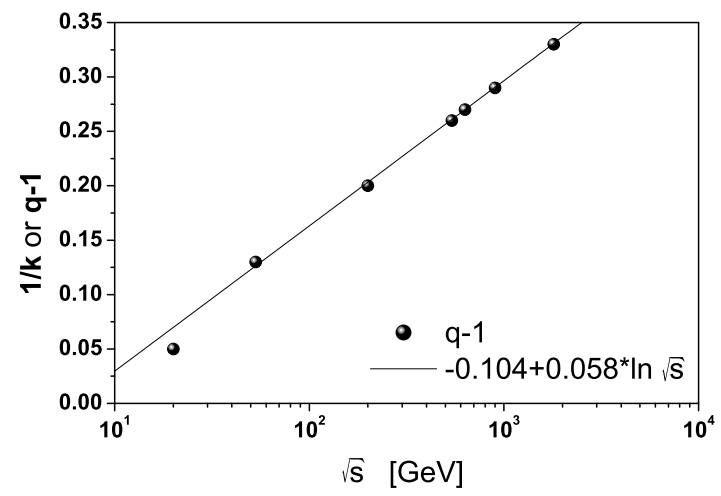

Fig. 3. The values of the nonextensivity parameter $q$ obtained in fits shown in the left panel of Fig. 2 compared with the values of the parameter $k$ of a Negative Binomial distribution fit to the corresponding multiplicity distributions as given in [1]. ( Reprinted Fig. 6 with permission from F.S. Navarra, O.V. Utyuzh, G. Wilk and Z. Włodarczyk, Phys. Rev. D 67, 114002 (2003). Copyright (2003) by the American Physical Society; URL http://link.aps.org/abstract/PRD/v67/e114002; DOI: 10.1103/ PhysRevD.67.114002.).

used only its mean value, $\langle N\rangle$, as given by experiment where $\langle N\rangle=\sum N P(N)$ with $P(N)$ being the multiplicity distribution 6 . On the other hand, the parameter $T$ in this case is not so much a temperature, but only a kind of "partition temperature", understood as mean energy per produced particle, i.e., $T \sim W /\langle N\rangle$ (where $W=K \sqrt{s}$, where $\sqrt{s}$ is the total energy of collision) 44. Therefore

\footnotetext{
6 Actually, we have used only its charged part, $\left\langle n_{c h}\right\rangle$, assuming that $N=\frac{3}{2}\left\langle n_{c h}\right\rangle$, i.e., neglecting in addition also possible fluctuations between the number of charged and neutral secondaries.
}

in this case one can just as well speak about the fluctuations of $\langle N\rangle$. Following therefore the ideas of [11] we would like to draw attention to the fact that the value of $k^{-1}$ may also be understood as the measure of fluctuations of the mean multiplicity (for example, in the usual Poissonian multiplicity distribution characterized just by a single parameter, the constant mean multiplicity $\bar{n}$ ), and in the case when such fluctuations are given by a gamma distribution with normalized variance $D(\bar{n})$, one obtains the Negative Binomial multiplicity distribution with

$$
\frac{1}{k}=D(\bar{n})=\frac{\sigma^{2}(\bar{n})}{\langle\bar{n}\rangle^{2}} .
$$

This is because in this case one has 45]:

$$
\begin{aligned}
P(n) & =\int_{0}^{\infty} d \bar{n} \frac{e^{-\bar{n}} \bar{n}^{n}}{n !} \cdot \frac{\gamma^{k} \bar{n}^{k-1} e^{-\gamma \bar{n}}}{\Gamma(k)} \\
& =\frac{\Gamma(k+n)}{\Gamma(1+n) \Gamma(k)} \cdot \frac{\gamma^{k}}{(\gamma+1)^{k+n}},
\end{aligned}
$$

where $\gamma=\frac{k}{\langle\bar{n}\rangle}$.

Therefore the situation in longitudinal phase space is following: When there are only statistical fluctuations in the hadronizing system one expects a Poissonian form of the corresponding multiplicity distributions. The existence of intrinsic (dynamical) fluctuations means that one allows the mean multiplicity $\bar{n}$ to fluctuate. It is natural to assume that these fluctuations contribute predominantly to the longitudinal phase space, i.e., that $D(\bar{n})=q-1$ and that

$$
q=1+\frac{1}{k}
$$

This is observed in the data.

The right hand panel of Fig. 2] displays results for $e^{+} e^{-}$ annihilations for which, by definition, $K_{q}=1$ (because always all the energy of initial leptons is available for the production of secondaries) and which can be fitted only with $q<1$ (in our case $q=0.6$ ). This should be contrasted with results obtained describing the $p_{T}$ distributions instead where one finds $q>123$. This point deserves closer scrutiny. The result for $q=1$ clearly shows that observed discrepancies are not connected with the particular value of $q$, but rather with some additional mechanisms operating here, the action of which would, however, change our results only slightly (for example, a possibility of two rather than one source or $y$-dependent $\left\langle p_{T}\right\rangle$, as mentioned already in [6]). With the above reservations, let us then take a closer look at the possible origin of $q<1$. We have already encountered a similar situation when in 13 . $q<1$ was simply closing the allowed a priori phase space, acting therefore as inelasticity parameter $K$. When considered as a signal of fluctuations (similar to the $q>1$ case) [12] it causes trouble because in this case the temperature $T$ does not reach an equilibrium state, in fact one now has that the source term (right hand side of Eq. (11)) is $T_{0} / \tau-(q-1) E / \tau$ rather than $T_{0} / \tau$ used for for $q>1$ case (cf., [12]). This means than that in this case we have a kind of dissipative transfer of energy from the 
region where (due to fluctuations) the temperature $T$ is higher. It could be any kind of convection-type flow of energy; for example, it could be connected with the emission of particles from this region (for example, in our case from a quark $(q)$ and antiquark $(\bar{q})$ jets formed in the first $e^{+} e^{-} \rightarrow q+\bar{q}$ to gluons and $q \bar{q}$ pairs and later on to finally observed hadrons). This means that $q<1$ signals that in the reaction considered, where $K_{q}=1$ and where we have to account for the whole energy exactly, conservation laws start to be important and there is no possibility for a stationary state with constant final temperature to develop. Instead, the temperature $T$ depends on the energy 7 , and for large energies tends to zero (notice that in this case one has a limitation on the allowed energy of the produced secondaries: $E \leq T_{0} /(1-q)$ ). This is not the case for the $p_{T}$ distribution analysis 23 . because most $p_{T}$ are small and are not influenced by the conservation laws but instead reflect a kind of stationary state with $q>1$.
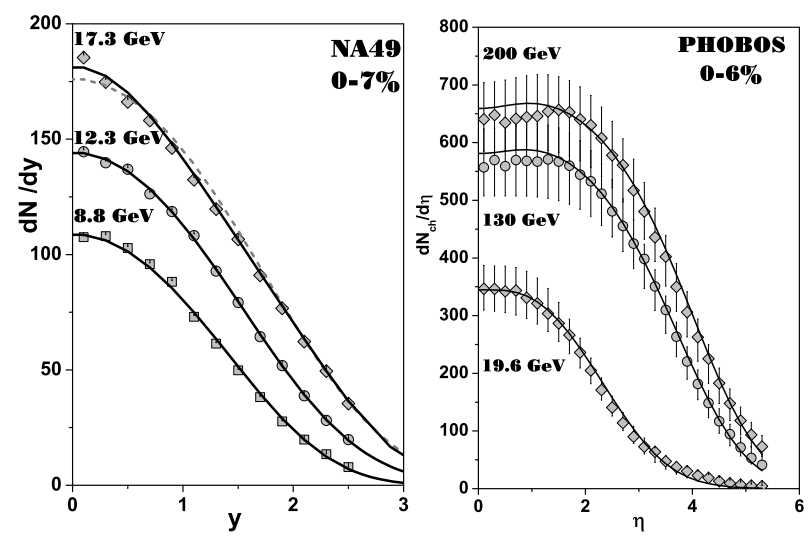

Fig. 4. Examples of applying a nonextensive approach to longitudinal distributions. Left panel: fits to NA49 data for $\mathrm{Pb}+\mathrm{Pb}$ collisions 42. Right panel: fits to PHOBOS data for $\mathrm{Au}+\mathrm{Au}$ collisions [43]. (Reprinted from Physica A340, F.S. Navarra, O.V. Utyuzh, G. Wilk and Z. Włodarczyk, "Information theory approach (extensive and nonextensive) to highenergy multiparticle production processes", 467, Copyright (2004), left panel, and from Physica A344, F.S. Navarra, O.V. Utyuzh, G. Wilk and Z. Włodarczyk, "Information theory in high-energy physics (extensive and nonextensive approach)", 568, Copyright (2004), right panel; both with permission from Elsevier; http://www.elsevier.com.).

Now look at the left hand panel of Fig. 4 It shows fits to NA49 data [42 on $\pi^{-}$production in $\mathrm{PbPb}$ collisions at three different energies per nucleon. The obtained values of nonextensivities and the corresponding inelasticities, $\left(q ; K_{q}=3 \cdot K_{q}^{\pi^{-}}\right)$are: $(1.2 ; 0.33)$ for $17.3 \mathrm{GeV}$, $(1.164 ; 0.3)$ for $12.3 \mathrm{GeV}$ and $(1.04 ; 0.22)$ for $8.6 \mathrm{GeV}$. The origin of $q>1$ in this case is not yet clear. The inelasticity seems to grow with energy. It is also obvious that, for higher energies, some new mechanism starts to operate because we cannot obtain agreement with data using only

\footnotetext{
7 Actually, in the case considered in 12 fluctuations depend on energy in the same way leaving the relative variance $\omega$ constant and leading to $q=1-\omega$.
}

energy conservation. The best fit for $17.3 \mathrm{GeV}$ for NA49 data actually for the case of $q=1$ and two sources separated in rapidity by $\Delta y=0.83$ (cf., 6] for other details).

Finally, the right hand panel of Fig. 4 presents fits to pion production in $\mathrm{Au}+\mathrm{Au}$ collisions $[43$ for the most central events (covering collisions proceeding with impact parameter range $0-6 \%) 8$. They can be fitted by choosing $K_{q}=1$ and then $q=1.29,1.26$ and 1.27 for energies 19.6, 130 and $200 \mathrm{GeV}$, respectively (cf., 20 for other details). As before, the origin of $q>1$ in this case is not yet clear.

Although the situation in AA collisions is not yet clear, we are quite confident that interpretation of the $q$ parameter offered here remains valid. But, before settling this, one point has to be addressed. Namely, the above $q$ were in fact $q_{L}$ responsible for the longitudinal dynamics only. On the other hand, multiplicity distributions are sensitive to $p=\sqrt{p_{L}^{2}+p_{T}^{2}}$ and, as we have seen here, both $p_{L}$ and $p_{T}$ show traces of fluctuations by leading to $q>1$. However, as we shall see below, $\left(q_{T}-1\right)<<\left(q_{L}-1\right)$ (what fits nicely the fact that $p_{T}$ space is very limited in comparison to $p_{L}$ one). Because there are no data measuring $p_{T}$ distributions at all values of rapidity $y$, i.e., providing correlations between parameters $(T ; q)=\left(T_{L} ; q_{L}\right)$ for longitudinal momenta (rapidity) distributions and $(T ; q)=\left(T_{T} ; q_{T}\right)$ for transverse momenta distributions, we offer only the following approximate answer. Noticing that $q-1=\sigma^{2}(T) / T^{2}$ (i.e., it is given by fluctuations of total temperature $T$ ) and assuming that $\sigma^{2}(T)=\sigma^{2}\left(T_{L}\right)+\sigma^{2}\left(T_{T}\right)$, one can estimate that the resulting values of $q$ should not be too different from

$$
q=\frac{q_{L} T_{L}^{2}+q_{T} T_{T}^{2}}{T^{2}}-\frac{T_{L}^{2}+T_{T}^{2}}{T^{2}}+1,
$$

which, for $T_{L} \gg T_{T}$, as is in our case, leads to the result that $q \sim q_{L}$, i.e., it is given by the longitudinal (rapidity) distributions only.

\subsection{Transverse phase space}

As discussed before, transverse phase space seems to be mainly dominated by the thermodynamical-like effects governed by the temperature $T$ [1]. It is therefore the best place too look for any fluctuations of temperature, i.e., to look for any deviation of the the inverse slope of transverse momenta distributions, $d N / d p_{T}$, from an exponential shape. That such deviations are really observed is seen in Fig. 5. On the left hand panel we can see fits to $p_{T}$ spectra measured by the UA1 experiment [4] in $p \bar{p}$ at different energies using Tsallis distribution, Eq. (5), with $X / \lambda \rightarrow$ $p_{T} / T$ and with the following values of $\left(T=T_{T}[\mathrm{GeV}], q=\right.$

8 Actually these data are originally presented not for the rapidity $y$ defined by the energy $E$ and the the longitudinal momentum $p_{L}$ but for the so called pseudorapidity $\eta$ defined by the total momentum $p$ and the longitudinal momentum $p_{L}$ instead. There is therefore some ambiguity when transferring them from $\eta$ to $y$ because of the pour knowledge of the rapidity dependence of the mean transverse momentum needed for such operation. 
$\left.q_{T}\right):(0.134,1.095),(0.135,1.105)$ and $(0.14,1.11)$ for energies 200, 500 and $900 \mathrm{GeV}$, respectively (the values of the parameter $q$ obtained in analysis of transverse momenta in elementary $e^{+} e^{-}$reaction is similar 23). These values should be compared with the corresponding values of $\left(T=T_{L} ; q=q_{L}\right)$ previously observed for rapidity distributions, which are equal to, respectively: $(11.74,1.2)$, $(20.39,1.26)$ and $(30.79,1.29)$ at comparable energies, cf. [19.
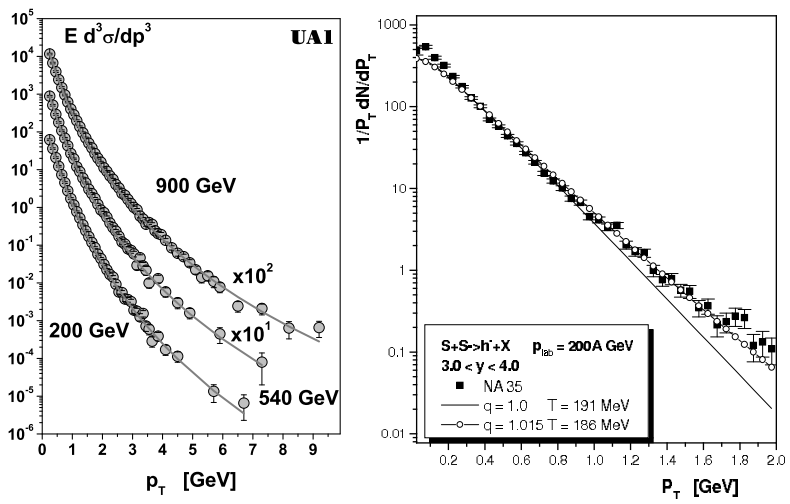

Fig. 5. Examples of applying a nonextensive approach to transverse momenta distributions. Left panel: fits to $p_{T}$ spectra from $p \bar{p}$ UA1 experiment [46] for different energies (see text for details (reprinted from Physica A340, F.S. Navarra, O.V. Utyuzh, G. Wilk and Z. Włodarczyk, "Information theory approach (extensive and nonextensive) to high-energy multiparticle production processes", 467, Copyright (2004), reproduced by permission of Elsevier, http://www.elsevier.com.). Right panel: fits to $S+S$ data from [47] (reproduced by permission of IOP Publishing Ltd from [15]).

The right hand panel of Fig. 5 shows an example of similar behavior observed for nuclear collisions. Such collisions are of special interest as they are the only place where a new state of matter, the Quark Gluon Plasma, can be produced 2 and, because of this, they are intensively investigated using a nonextensive approach (see, for example, $12,21,24,25,28)$ ). As one can see, the best fit is obtained for $q>1$, albeit in this case the value of $(q-1)$, which is the real measure of fluctuations, is noticeably smaller than in the case of elementary reactions mentioned above. On the other hand, although very small $(|q-1| \sim 0.015)$, this deviation leads to a quite substantial relative fluctuations of the temperature existing in the nuclear collisions, namely one gets that $\Delta T / T \simeq 0.12$.

The question then arises: if this is treated seriously, what we are really measuring, what physical observable does it correspond to? It is important to stress that these are fluctuations existing in small parts of a hadronic system in respect to the whole system rather than of the event-by-event type for which,

$$
\Delta T / T=0.06 / \sqrt{N} \rightarrow 0
$$

for large $N$. The answer is that the measured fluctuations provide a direct measure of the total heat capacity $C$ of the system [48],

$$
\frac{\sigma^{2}(\beta)}{\langle\beta\rangle^{2}}=\frac{1}{C}=\omega=q-1,
$$

( $\beta=\frac{1}{T}$ ) in terms of $\omega=q-1$. Therefore, single particle distributions of produced secondaries, if only measured very precisely, can a priori provide us information not only on the temperature $T$ of the hadronizing system but also, when investigated using a nonextensive approach, give us information (via value of $q-1$ ) on its total heat capacity $C$. In this way one can not only check whether some (approximate) thermodynamical state is formed in a single collision but also what are its thermodynamical properties - a very important feature, especially in what concerns the existence and type of the possible phase transitions [2].
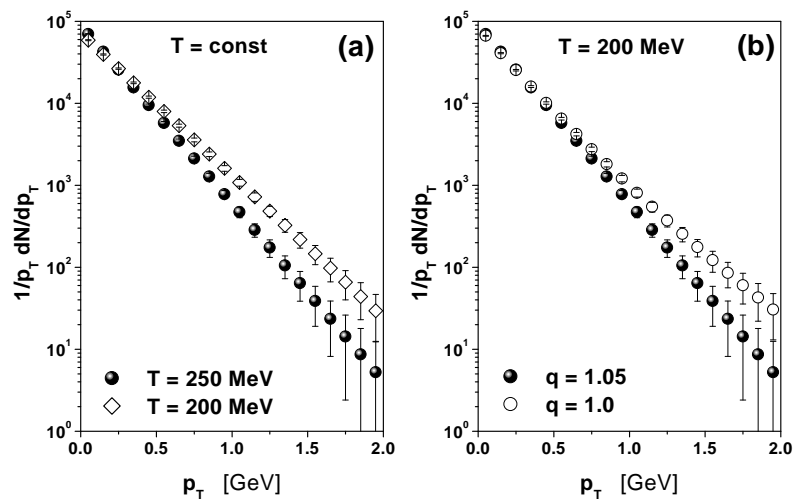

Fig. 6. (a) Normal exponential $p_{T}$ distributions i.e., $q=1$ ) for $T=200 \mathrm{MeV}$ (black symbols) and $T=250 \mathrm{MeV}$ open symbols). (b) Typical event from central $P b+P b$ at $E_{\text {beam }}=$ $3 A \cdot \mathrm{TeV}$ for $=200 \mathrm{MeV}$ for $q=1$ (black symbols) exponential dependence and $q=1.05$ (open symbols). (Reprinted from Physica A305, G. Wilk and Z. Włodarczyk, "Application of nonextensive statistics to particle and nuclear physics", 227, Copyright (2002), reproduced by permission of Elsevier, http://www.elsevier.com ).

The next question is: how plausible is such a program? The point is, as discussed above, that one performs fits using $T$ and $q$ in a Tsallis distributions rather than only $T$ in the usual exponential ones. However, the corresponding data on $p_{T}$ are effectively integrated over the longitudinal phase space (or, at least a part of it) and are averaged over many events. The best thing would be to observe such an effect in a single events, then an event-by-event analysis of data would be possibly. Fig. 6 shows what we can expect. Two scenarios are demonstrated there: $(a) T$ is constant in each event but (because, for example, of different initial conditions) it fluctuates from event to event and $(b)$ $T$ fluctuates in each event around some mean value $T_{0}$. We have chosen for comparison a typical event obtained in simulations performed for central $P b+P b$ collisions taking place for beam energy equal $E_{\text {beam }}=3 A \cdot \mathrm{TeV}$ (expected shortly in ALICE experiment at LHC). Density of particles in the central region (defined by rapidity window $-1.5<y<1.5)$ is chosen to be equal to $\frac{d N}{d y}=6000$. 
In case $(a)$ in each event one expects exponential dependence with $T=T_{\text {event }}$ and possible departure from it would occur only after averaging over all events. It would reflect fluctuations originating from different initial conditions for each particular collision. This situation is illustrated in Fig. 6] (a) where $p_{T}$ distributions for $T=200$ $\mathrm{MeV}$ (black symbols) and $T=250 \mathrm{MeV}$ (open symbols) are presented. Such values of $T$ correspond to typical uncertainties in $T$ expected at $\mathrm{LHC}$ accelerator at CERN. Notice that both curves presented here are straight lines. In case $(b)$ one should observe departure from the exponential behavior already on the single event level and it should be fully given by $q>1$. This reflects a situation when, due to some intrinsically dynamical reasons, different parts of a given event can have different temperatures, as we have discussed above. In Fig. 6 (b) black symbols represent exponential dependence obtained for $T=200$ $\mathrm{MeV}$ (the same as in Fig. 6 (a) ), open symbols show the power-like dependence as given by (5) with the same $T$ and with $q=1.05$ (notice that the corresponding curve bends slightly upward here). In this typical event we have $\sim 18000$ secondaries, i.e., practically the maximal possible number. Notice that here points with highest $p_{T}$ already correspond to single particles. As one can see, experimental differentiation between these two scenarios will be very difficult, although not totally impossible. On the other hand, if successful it would be very rewarding 9 .

The following remarks are worth to be done at this point.

- We are using rather freely the notion of fluctuating temperature. The question then arises whether is makes sense. Not going into a detailed dispute, we would only like to mention at this point that traces of this idea can already be found in 49,50, 10. In particular it is important when discussing some peculiarities of the phase diagrams, which are important when addressing the question of possible phase transitions between QCD and normal matter 50. What we want to do is to bring to ones attention the fact that event-by-event analysis allows us (at least in principle) to detect fluctuations of temperature taking place in a given event. This is more than an indirect measure of fluctuations of $T$ proposed some time ago in 52 or more direct fluctuations of $T$ from event to event discussed in [49].

- As the heat capacity $C$ is proportional to the volume, $C \propto V$, in our case $\mathrm{V}$ would be the volume of the interaction (or hadronization), it is expected to grow with volume and, respectively, $q$ is expected to decrease with $V$. This is indeed the case if one puts together the results for $e^{+} e^{-}, p p$ and $p \bar{p}$ and and $A A$ collisions for example, those of [23] for $e^{+} e^{-}$collisions, together

\footnotetext{
${ }^{9}$ It is interesting to realize that for the Planckian gas at $T=186 \mathrm{MeV}$, occupying volume of the order of the volume of sulfur nucleus, one gets $C=34.4$ per degree of freedom, which leads, using Eq. (20), to $q=1.015$ obtained for such system for the $p_{T}$ dependence of produced secondaries.

10 For those interested in discussion on the problem of internal consistency (or inconsistency) of the notion of fluctuations of temperature in thermodynamics we refer to [4, 49, 51].
}

with those of [6] for $p \bar{p}$ collisions and all results for heavy-ion collisions, like [24,20,28, and especially [21. where such a trend was found when analyzing heavyion events with different centrality (i.e., with different volumes $V$ ).

- As the parameter $q$ replaces in some sense the action of many not yet identified dynamical factors, one expects that, with such factors included, $q$ should diminish. This is precisely what has been demonstrated in 21 analyzing transverse momenta of pions produced in RHIC experiments by using a simple minded Tsallis formula and an accordingly modified Hagedorn [53] approach which already contains in it some dynamics (based on a special bootstrap hypothesis of resonances composed out of resonances itd.). In the second case the values of $q-1$ found are much smaller, but still remain nonzero indicating therefore the existence of some residual additional dynamic there.

- As demonstrated in 21], using a nonextensive version of the statistical model allows us to describe data well in the domain previously believed to be governed entirely be pure jet physics. Deviations (i.e., dominance of truly hard collisions) start at $p_{T}$ near $10 \mathrm{GeV}$ and further. It would mean that the so called mini-jet region can probably also be investigated using a nonextensive approach (what should be, however, checked in more detail int the future).

\subsection{The whole phase space}

Already presenting results for the longitudinal phase space we encountered multiplicity distributions of produced secondaries. They involve the whole of phase space, both its longitudinal and transverse components. However, as we have already stressed, because $\left(q_{L}-1\right)>>\left(q_{T}-1\right)$, the dominant role of the longitudinal dynamic in establishing the actual number of produced secondaries and its fluctuation from event to event is obvious. We shall now discuss this problem in more detail.

Previous findings could be summarized in the following way: knowing the amount of energy $W$ which is going to be transferred to the produced secondaries (i.e., knowing the inelasticity $K$ of reaction [39]) and the mean number of produced secondaries, $\langle N\rangle$, and respecting the fact that they are essentially distributed in the longitudinal phase space only, one arrives, after using the information theory approach (cf., 5), with the usual exponential distribution in $E=\left\langle m_{T}\right\rangle \cosh y$. Additional information on the fact that produced secondaries are distributed not according to a Poisson distribution but rather according to NB distribution characterized by parameter $k$ is enough to get $q$-exponential distribution in $E$ with $q=1+1 / k$.

Now, it turns out that the opposite is also true, namely, as we have shown in [22], the fact that $N$ particles are distributed in energy via $N$-particle Tsallis distribution described by the nonextensivity parameter $q$ allows us to show that their number distribution has to be of the NB type with $k=1 /(q-1)$. To illustrate this we first start with the derivation of Poisson multiplicity distribution 
and then to compare it with the corresponding derivation of the NB distribution 22 .

\subsubsection{Poisson multiplicity distribution}

This distribution arises in a situation where in some process one has $N$ independently produced secondaries with energies $\left\{E_{1, \ldots, N}\right\}$, each distributed according to the simple Boltzmann distribution:

$$
f\left(E_{i}\right)=\frac{1}{\lambda} \cdot \exp \left(-\frac{E_{i}}{\lambda}\right)
$$

(where $\lambda=\langle E\rangle$ ). The corresponding joint probability distribution is then given by:

$$
f\left(\left\{E_{1, \ldots, N}\right\}\right)=\frac{1}{\lambda^{N}} \cdot \exp \left(-\frac{1}{\lambda} \sum_{i=1}^{N} E_{i}\right)
$$

For independent energies $\left\{E_{i=1, \ldots, N}\right\}$ the sum $E=\sum_{i=1}^{N} E_{i}$ is then distributed according to the following gamma distribution,

$$
g_{N}(E)=\frac{1}{\lambda(N-1) !} \cdot\left(\frac{E}{\lambda}\right)^{N-1} \cdot \exp \left(-\frac{E}{\lambda}\right),
$$

distribuant of which is

$$
G_{N}(E)=1-\sum_{i=1}^{N-1} \frac{1}{(i-1) !} \cdot\left(\frac{E}{\lambda}\right)^{i-1} \cdot \exp \left(-\frac{E}{\lambda}\right) .
$$

Eq. (23) follows immediately either by using characteristic functions or by sequantially performing integration of the joint distribution (22) and noticing that:

$$
g_{N}(E)=g_{N-1}(E) \frac{E}{N-1} .
$$

For energies such that

$$
\sum_{i=0}^{N} E_{i} \leq E \leq \sum_{i=0}^{N+1} E_{i}
$$

the corresponding multiplicity distribution has a Poissonian form (notice that $E / \lambda=\langle N\rangle$ ):

$$
\begin{aligned}
P(N) & =G_{N+1}(E)-G_{N}(E)= \\
& =\frac{\left(\frac{E}{\lambda}\right)^{N}}{N !} \cdot \exp (-\alpha E)=\frac{\langle N\rangle^{N}}{N !} \cdot \exp (-\langle N\rangle)
\end{aligned}
$$

In other words, whenever we have variables $E_{1, \ldots, N, N+1, \ldots}$ taken from the exponential distribution $f\left(E_{i}\right)$ and whenever these variables satisfy the condition $\sum_{i=0}^{N} E_{i} \leq E \leq$ $\sum_{i=0}^{N+1} E_{i}$, then the corresponding multiplicity $N$ has a Poissonian distribution 11 .

\footnotetext{
11 Actually, this is the method of generating Poisson distribution in the numerical Monte-Carlo codes.
}

\subsubsection{Negative Binomial multiplicity distribution}

This distribution arises when in some process $N$ independent particles with energies $\left\{E_{1, \ldots, N}\right\}$ which are now distributed according to Tsallis distribution,

$$
h\left(\left\{E_{1, \ldots, N}\right\}\right)=C_{N}\left[1-(1-q) \frac{\sum_{i=1}^{N} E_{i}}{\lambda}\right]^{\frac{1}{1-q}+1-N}
$$

with normalization constant $C_{N}$ given by

$$
\begin{aligned}
C_{N} & =\frac{1}{\lambda^{N}} \prod_{i=1}^{N}[(i-2) q-(i-3)]= \\
& =\frac{(q-1)^{N}}{\lambda^{N}} \cdot \frac{\Gamma\left(N+\frac{2-q}{q-1}\right)}{\Gamma\left(\frac{2-q}{q-1}\right)} .
\end{aligned}
$$

It means that there are some intrinsic (so far unspecified but summarily characterized by the parameter $q$ ) fluctuations present in the system under consideration. In this case we do not know the characteristic function for the Tsallis distribution, however, because we are dealing here only with variables $\left\{E_{i=1, \ldots, N}\right\}$ occurring in the form of the sum, $E=\sum_{i=1}^{N} E_{i}$, one can still sequentially perform integrations of the joint probability distribution (28) and, noting that (as before, cf. eq. (25) )

$$
h_{N}(E)=h_{N-1}(E) \frac{E}{N-1}=\frac{E^{N-1}}{(N-1) !} h\left(\left\{E_{1, \ldots, N}\right\}\right),
$$

we arrive at formula corresponding to eq. (23), namely

$$
\begin{aligned}
h_{N}(E) & =\frac{E^{(N-1)}}{(N-1) ! \lambda^{N}} \times \\
\times & \prod_{i=1}^{N}[(i-1) q-(i-3)]\left[1-(1-q) \frac{E}{\lambda}\right]^{\frac{1}{1-q}+1-N}
\end{aligned}
$$

with distribuant given by

$$
\begin{aligned}
H_{N}(E) & =1-\sum_{j=1}^{N-1} \tilde{H}_{i}(E) \quad \text { where } \\
\tilde{H}_{i}(E) & =\frac{E^{i-1}}{(j-1) ! \lambda^{j}} \times \\
\times & \prod_{i=1}^{j}[(i-1) q-(i-3)]\left[1-(1-q) \frac{E}{\lambda}\right]^{\frac{1}{1-q}+1-j} .
\end{aligned}
$$

As before, for energies $E$ satisfying the condition given by eq. (26), the corresponding multiplicity distribution is equal to

$$
P(N)=H_{N+1}(E)-H_{N}(E)
$$

and is given by the Negative Binomial distribution :

$$
P(N)=\frac{(q-1)^{N}}{N !} \cdot \frac{q-1}{2-q} \cdot \frac{\Gamma\left(N+1+\frac{2-q}{q-1}\right)}{\Gamma\left(\frac{2-q}{q-1}\right)} \times
$$




$$
\begin{aligned}
& \times\left(\frac{E}{\lambda}\right)^{N}\left[1-(1-q) \frac{E}{\lambda}\right]^{-N+\frac{1}{1-q}}= \\
& =\frac{\Gamma(N+k)}{\Gamma(N+1) \Gamma(k)} \cdot \frac{\left(\frac{\langle N\rangle}{k}\right)^{N}}{\left(1+\frac{\langle N\rangle}{k}\right)^{N+k}},
\end{aligned}
$$

where the mean multiplicity and variance are, respectively,

$$
\begin{aligned}
\langle N\rangle & =\frac{E}{\lambda} \\
\operatorname{Var}(N) & =\frac{E}{\lambda}\left[1-(1-q) \frac{E}{\lambda}\right]=\langle N\rangle+\langle N\rangle^{2} \cdot(q-1) .
\end{aligned}
$$

This distribution is defined by the parameter $k$ equal to:

$$
k=\frac{1}{q-1} .
$$

Notice that for $q \rightarrow 1$ one has $k \rightarrow \infty$ and $P(N)$ becomes a Poisson distribution, whereas for $q \rightarrow 2$ one has $k \rightarrow 1$ and we are obtaining geometrical distribution 12 .

\section{Further developments}

Let us now proceed a step further in Eq. (10) by writing it in the following form,

$$
c_{p} \rho \frac{\partial T}{\partial t}=a\left(T^{\prime}-T\right)+\eta f(u)
$$

with a new term, $\eta f(u)$, which presents the effect of a possible viscosity (with viscosity coefficient $\eta$ ) existing in the system. The function $f(u)$ contains terms dependent on the velocity in the form of $\frac{\partial u_{i}}{\partial x_{k}}-\frac{\partial u_{k}}{\partial x_{l}}$. Using as before $T^{\prime}$ defined by (7) we get an extension of Eq. (11):

$$
\begin{aligned}
\frac{\partial T}{\partial t}+\left[\frac{1}{\tau}+\xi(t)\right] T & =\frac{1}{\tau} T_{0}+\eta f(u) \frac{1}{c_{p} \rho}= \\
& =\frac{1}{\tau}\left[T_{0}+\frac{\eta \tau}{c_{p} \rho} f(u)\right] .
\end{aligned}
$$

This equation leads to the Langevin equation resulting in fluctuations of the temperature $T$ given in the same form of Eq. (12) as before but with

$$
\mu=\frac{1}{q-1}\left[T_{0}+\frac{\eta \tau}{c_{p} \rho} f(u)\right]=\frac{T_{e f f}}{q-1} .
$$

In this way previous $T_{0}=\langle T\rangle$ has now been replaced by a kind of effective temperature

$$
T_{e f f}=T_{0}+\frac{\eta \tau}{c_{p} \rho} f(u)=T_{0}+\frac{\eta}{a} f(u) .
$$

\footnotetext{
12 Actually the parameter $k$ in NB can be simply expressed by the correlation coefficient $\rho$ for the two-particle energy correlations, $k=(\rho+1) / \rho$, see [22] for details.
}

Introducing a kinetic coefficient of conductance $\nu=\eta / \rho$ and denoting $\kappa=c_{p} / c_{V}$, where $c_{V}$ is specific heat under the constant volume for which $1 / c_{V}=q-1$, we have that

$$
T_{\text {eff }}=T_{0}+\frac{\nu \tau}{\kappa c_{V}} f(u)=T_{0}+(q-1) \frac{\nu \tau}{\kappa} f(u)
$$

or, because $\tau D=q-1$, one can write this also as

$$
T_{e f f}=T_{0}+(q-1)^{2} \frac{\nu}{\kappa D} f(u) .
$$

In 28] the transverse momentum spectra of pions and protons and antiprotons produced in the interactions of $\mathrm{P}+\mathrm{P}, \mathrm{D}+\mathrm{Au}$ and $\mathrm{Au}+\mathrm{Au}$ at $\sqrt{s_{N N}}=200 \mathrm{GeV}$ at RHIC-BNL [54] were analyzed using a nonextensive approach. Among other things they found dependencies of the nonextensivity parameter $q$ and temperature $T$ on the number of participants, $N_{p}$ (i.e., number of nucleons taking part in a given $A A$ collision in the production of secondaries). From them we have obtained a dependence of $T$ on the parameter $q$ which are shown in Figs. 7 and 8. In all cases we find that $f(u)<0$ and that $T$ seems to be linearly dependent on $q-1$.

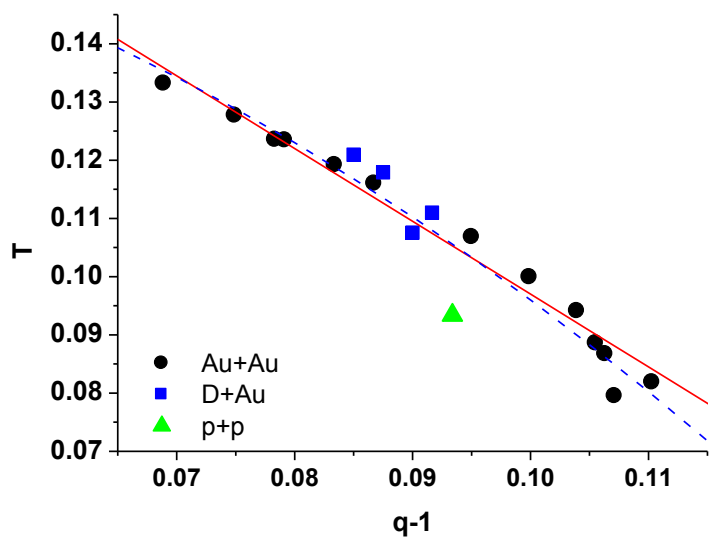

Fig. 7. Dependence of temperature $T$ (in $\mathrm{GeV}$ ) on the parameter $q$ for production of negative pions in different reactions. The solid line shows a linear fit to obtained results: $T=0.22-1.25(q-1)$ (cf., Eq. (41)) and dashed line shows the corresponding quadratic fit: $T=0.17-7.5(q-1)^{2}$ (cf., Eq. (42)).

These results can be compared with old results for $e^{+} e^{-}$annihilation reactions discussed some time ago in terms of $q$-statistics in [23. The $q$ dependence of the temperature parameter $T$ which can be deduced from them is shown in Fig. 9. Notice that now the temperature is lower and depends only weakly on $q$.

Finally, let us discuss results on fluctuations of multiplicity observed in heavy-ion collisions 55. They exhibit non-monotonic changes as function of the number of participants $N_{p}$ [55]. Actually, also changes of $\langle N\rangle$ show nonlinear increase, though not so spectacular. Acting in the 


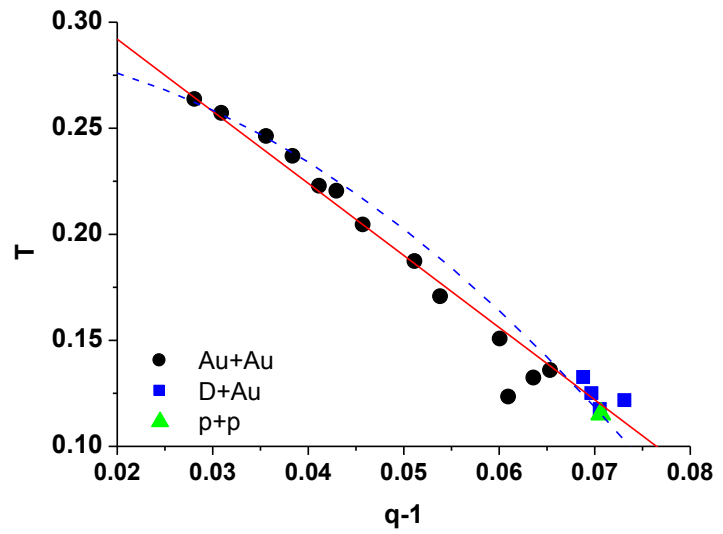

Fig. 8. The same as in Fig. 7 but for the produced antiprotons. The linear fit (solid line) is: $T=0.36-3.4(q-1)$ whereas quadratic one (dashed line) is: $T=0.29-35(q-1)^{2}$.

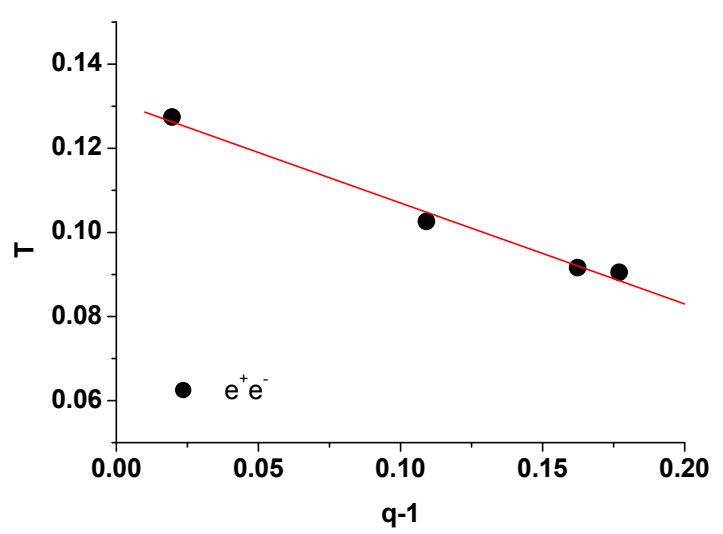

Fig. 9. Dependence of temperature $T$ (in $\mathrm{GeV}$ ) on the parameter $q$ for the production of pions in $e^{+} e^{-}$annihilation reactions. $T=0.131-0.24(q-1)$.

spirit of our analysis here we can expect that

$$
\frac{\frac{\operatorname{Var}(N)}{<N>}-1}{<N>}=q-1,
$$

but now, with $T_{\text {eff }}$ we can show that

$$
<N>=\frac{W}{T_{e f f}}
$$

where $T_{\text {eff }}$ is given by Eq. (41) and where $W$ is the full accessible energy. We have therefore that

$$
\frac{\langle N\rangle-n_{0} N_{p}}{<N>}=c(q-1) .
$$

Here $n_{0}$ is the multiplicity in the single nucleon-nucleon collision measured in the region of acceptance, $c=-\frac{\nu \tau}{\kappa} \frac{f(u)}{T_{0}}$ (notice that $c$ is positive because, as was found from Figs. 7 and $8, f(u)<0)$.

In Figs. 10 and 11 we show what can be extracted from $P b P b$ collision data taken by NA49 experiment [55]. As one can observe the data confirm our expectation that

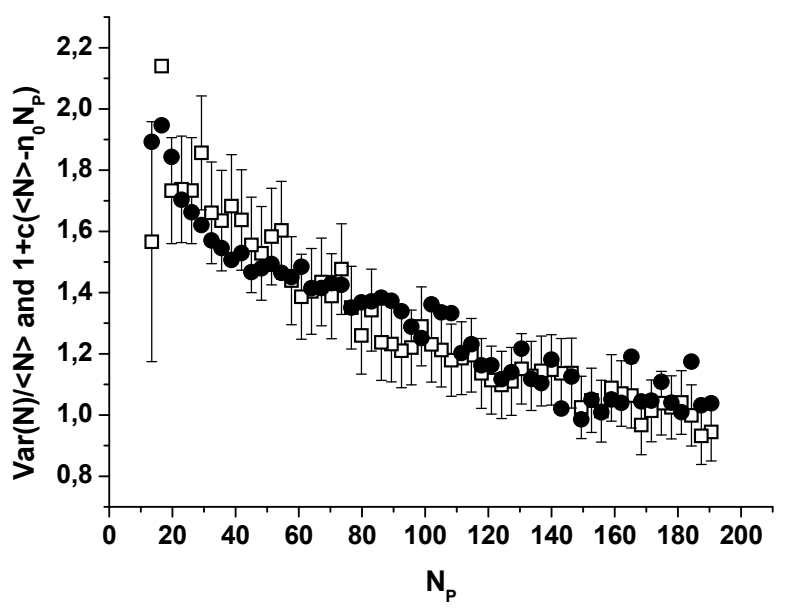

Fig. 10. Comparison of $\operatorname{Var}(N) /\langle N\rangle$ versus $N_{p}$ (squares) with $1+c\left(\langle N\rangle-n_{0} N p\right)$ vs $N_{p}$ (circles) (here $n_{0}=0.642$ and $c=4.1$. Data are for negatively charged particles from $\mathrm{PbPb}$ collisions as collected by NA49 experiment [55].

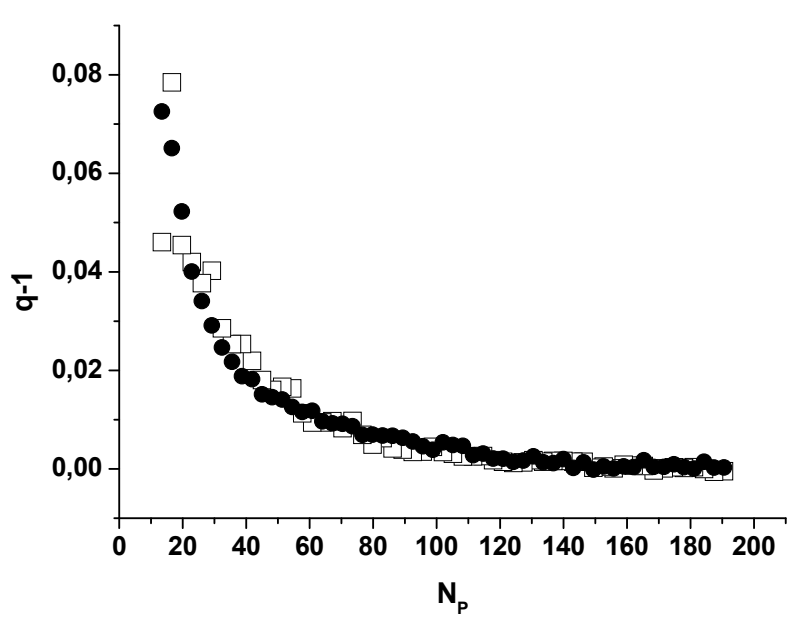

Fig. 11. The same as in Fig. 10 but translated to $q-1$ vs $N_{p}$. Squares were obtained from $\operatorname{Var}(N) /\langle N\rangle$ vs $N_{p}$ and circles from $\langle N\rangle$ vs $N_{p}$. As before $n_{0}=0.643$ and $c=4.1$.

dependencies of $\operatorname{Var}(N) /\langle N\rangle$ and $\langle N\rangle$ on the number of participants $N_{p}$ are, after introducing the concept of $T_{\text {eff }}$, essentially the same. The value of $n_{0}=0.642$ is also sensible, being only a little greater than the multiplicity observed in $p p$ collisions when calculated using the acceptance of the NA49 experiment. Notice also that value of $c=4.1$ obtained here for $\mathrm{PbPb}$ collisions is not far from the value $1.25 / 0.22=5.7$ obtained for data from RHIC (i.e., for $A u A u$ collisions but at much higher energy) which we have obtained in Fig. 7

We close this part by noticing that this problem is not trivial since none of the known models for multiparticle production processes describes $\operatorname{Var}(N) /\langle N\rangle$ vs $N_{p}$ observed experimentally [55]. They are described only by some specialized models addressing fluctuations, like the percolation model [56, the model assuming inter-particle 
correlations caused by the combination of strong and electromagnetic interactions [57] or the transparency, mixing and reflection model 58. Actually, all those attempts were addressing only $\operatorname{Var}(N) /\langle N\rangle$ vs $N_{p}$ but not $\langle N\rangle$ vs $N_{p}$. From this perspective, results presented above in Figs. 10 and 11 confirm the reasonableness of the idea of $T_{\text {eff }}$ introduced in this Section. If one uses $\operatorname{Var}(N) /\langle N\rangle$ vs $N_{p}$ to obtain $q-1$ then it turns out that the same value of $q-1$ describes also dependence of $\langle N\rangle$ on $N_{p}$; this can only be using $T_{\text {eff }}$ and this is because it depends on $q-1$.

\section{Remarks and summary}

Let us start with two remarks which are in order here:

(i) Results which recall directly to Tsallis entropy were obtained using the constraint $\sum p_{i}=1$ and the formula $\sum_{i} p_{i}^{q} A_{i}=\langle A\rangle_{q}$ for the $q$-expectation values. On the other hand, there exists a formalism, which expresses both the Tsallis entropy and the expectation values using the so-called escort probability distributions [59: $P_{i}=p_{i}^{q} / \sum_{i} p_{i}^{q}$. However, as was shown in 60], such an approach is different from the normal nonextensive formalism because the Tsallis entropy expressed in terms of the escort probability distributions has some difficulty with the property of concavity. From our limited point of view, it seems that there is no problem in what concerns practical, phenomenological applications of nonextensivity as discussed in the present work. Namely, using $P_{i}$ one gets distributions of the type $c[1-(1-q) x / l]^{q /(1-q)}$, which is, in fact, formally identical with $c[1-(1-Q) x / L]^{1 /(1-Q)}$, provided we identify: $Q=1+(q-1) / q, L=l / q$ and $c=(2-Q) / L=1 / l$. The mean value is now $\langle x\rangle=L /(3-2 Q)=l /(2-q)$ and $0<Q<1.5$ (to be compared with $0.5<q<2)$. Both distributions are identical and the problem, of which of them better describes data is artificial.

(ii) One should be aware that there is still an ongoing discussion on the meaning of the temperature in nonextensive systems. However, the small values of the parameter $q$ deduced from data in transverse phase space (where the connection with thermodynamical approach makes sense, as discussed before) allow us to argue that, to first approximation, $T$ can be regarded as the hadronizing temperature in such a system. One must only remember that in general what we study here is not so much the state of equilibrium but rather some kind of stationary state. For a thorough discussion of the temperature of nonextensive systems, see [61].

With the above reservations in mind, we can summarize that, when looking from the point of view of a statistical approach [1,2, the power-law behavior of many distributions observed in elementary and heavy ion collisions can be traced back to the necessity of using the nonextensive version of a statistical model (here taken in the form proposed by Tsallis [8]).

We interpret this as a sign of some intrinsic fluctuations present in any hadronizing system, which were only recently to be recognized as vital observable when searching for the production of the QGP form of matter [2]. In fact, a number of works 62 have demonstrated the existence in such reactions of event-by-event fluctuations of the average transverse momenta $\langle p\rangle$ per event. The quantities considered were: $\operatorname{Var}(\langle p\rangle) /\langle\langle p\rangle\rangle^{2}$ and $\left\langle\Delta p_{i} \Delta p_{j}\right\rangle /\langle\langle p\rangle\rangle^{2}$. These quantities can be shown $[22$ to be fully determined by $\omega$ as defined by Eq. (4), i.e., by fluctuations of the temperature $T$ of hadronizing system - a vital observable when searching for QGP ${ }^{13}$. In fact, when considering the case of $N_{e v}$ events with $N_{k}$ particles in the $k^{\text {th }}$ event, one has that

$$
\frac{\operatorname{Var}(\langle p\rangle)}{\langle\langle p\rangle\rangle^{2}}=\frac{\operatorname{Var}(T)}{\langle T\rangle^{2}}=\omega
$$

where

$$
\langle\langle p\rangle\rangle=\frac{1}{N_{e v}} \sum_{k}^{N_{e v}}\langle p\rangle_{k} ; \quad \text { with } \quad\langle p\rangle_{k}=\frac{1}{N_{k}} \sum_{i}^{N_{k}} p_{i}
$$

This is what we have shown in the last part od Section 3 This is the problem which needs further investigations.

We close with some remarks:

- Although our original investigations presented here were based on the notion of Tsallis entropy (usually with the help of information theory) one must mention that one can also get Tsallis distribution without resorting to a Tsallis entropy altogether (see, for example, 64]).

- The other way to get a Tsallis distribution from some general thermodynamical considerations was presented in 65. It is based on allowing a linear dependence of the temperature $T$ on energy, $T=T_{0}+(q-1) E$. Here temperature is not fluctuating. Actually, if one would like to follow this approach and to have Tsallis distribution with $T_{\text {eff }}$ discussed in Section 3 one should write $T=T_{0}+(q-1) \cdot$ const $+(q-1) E$. Then $T=T_{0}$ only would result in $\exp \left(-E / T_{0}\right), T=$ $T_{0}(q-1) E$ would result in the usual Tsallis distribution $\exp _{q}\left(-E / T_{0}\right), T=T_{\text {eff }}=T_{0}+(q-1) \cdot$ const would give $\exp \left(-E / T_{\text {eff }}\right)$ and, finally, $T=T_{\text {eff }}=$ $T_{0}+(q-1) \cdot$ const $+(q-1) E$ would give $\exp _{q}\left(-E / T_{e f f}\right)$.

- Notice that for $x>>\lambda /(q-1)$ Tsallis distribution becomes a pure power low and loses its dependence on the scale $\lambda: f(x) \sim[1-(1-q) x / \lambda]^{1 /(1-q)} \longrightarrow x^{1 /(1-q)}$.

- Instead of using an intrinsic fluctuations one can also obtain a power law distribution by using the notion of self-organized criticality 66 in a cascade processes (cf., [16,67]).

- Another interesting possibility, not yet fully explored, is that, as shown in 68, one can formulate a description of the so called stochastic networks using nonextensive information theory based on Tsallis statistics. Using this approach one can then demonstrate 69

$\overline{13}$ Generally speaking, an analysis of transverse momenta $p_{T}$ alone indicates very small fluctuations of $T$. On the other hand, as reported in 63], the measured fluctuations of multiplicities of produced secondaries are large (i.e., multiplicity distributions are substantially broader than Poissonian). 
that hadron production viewed as formation of a specific stochastic network can explain in a natural way the power-law distributions of transverse mass spectra of pions found in 70 .

- In the string models of production of hadrons the natural distribution in $p_{T}$ is $\exp \left(-\pi m_{T}^{2} / \kappa\right)$ rather than $\exp \left(m_{T} / T\right)$ (where $\kappa$ is string tension) really observed. However, if one allows for the gaussian fluctuations of the parameter $\kappa$ (characterized by parameter $\left\langle\kappa^{2}\right\rangle$ which can be connected with the fluctuations in the QCD vacuum) then the first form is transformed into the second one with $T=\sqrt{\left\langle\kappa^{2}\right\rangle /(2 \pi)}$ (i.e., in this approach parameter $T$ characterizes rather the properties of the QCD vacuum than those of hadrons) - see [71.

- Finally, recall that when applied to the hydrodynamical model of multiparticle production the nonextensivity approach converts the usual nonviscous hadronic fluid into the viscous one preserving, however, the usual linear flow equations (albeit now given in a nonextensive form) 72 .

Partial support (GW) of the Ministry of Science and Higher Education under contracts 1P03B02230 is acknowledged.

\section{References}

1. R. Hagedorn, Nuovo Cim. (Suppl.) 3, 147 (1965), Nuovo Cim. A 52, 64 (1967) and Riv. Nuovo Cim. 6 (1983); C. Geich-Gimbel, Int. J. Mod. Phys. A 4, 1527 (1989). U. Heinz, J. Phys. G 25, 263 (1999) 263; F. Becattini, Nucl. Phys. A 702, 336 (2002), F. Becattini, G. Passaleva, Eur. Phys. J. C 23, 551 (2002); W. Broniowski, A. Baran, W. Florkowski, Acta Phys. Polon. B 33, 4325 (2002) 4235.

2. Cf., QM2008 proceedings, J. Phys. G 35 (10) (2008); see also: B.Müller, Nucl. Phys. A 774, 433 (2006); M. Gyulassy and L. McLerran, ibid. A 750, 30 (2005); I. Vitev, Int. J. Mod. Phys. A 20, 3777 (2005); R. D. Pisarski, Braz. J. Phys. 36, 122 (2006) and references therein.

3. L. Van Hove, Z. Phys. C 21, 93 (1985) and C 27, 135 (1985).

4. B. Müller and J. L. Nagle, Annu. Rev. Nucl. Part. Sci. 56, 93 (2006) and references therein.

5. G. Wilk, Z. Włodarczyk, Phys. Rev. D 43, 794 (1991); O.V.Utyuzh, G.Wilk and Z.Włodarczyk, Acta Phys. Hung. A - Heavy Ion Phys. 25, 65 (2006).

6. F.S. Navarra, O.V. Utyuzh, G. Wilk and Z. Włodarczyk, Physica A 340, 467 (2004).

7. C. Arndt, Information measures - Information and its Description in Science and Engineering, Springer, 2004; T.I.J. Taneja, Generalized Information Measures and Their Applications, on-line book, http://www.mtm.ufsc.br/taneja/ book/book.html.

8. C. Tsallis, J. Stat. Phys. 52, 479 (1988), Braz. J. Phys. 29, 1 (1999), Physica A 340, 1 (2004) and Physica A 344, 718 (2004) and references therein. See also Nonextensive Statistical Mechanics and its Applications, S. Abe and Y. Okamoto (Eds.), Lecture Notes in Physics LPN560, Springer (2000) and Nonextensive Entropy - interdisciplinary applications, M. Gell-Mann and C. Tsallis (Eds.), (a volume in the Santa Fe Institute Studies in the Science of Complexity), Oxford University Press (2004). For an updated bibliography on this subject see http://tsallis.cat.cbpf.br/biblio.htm C. Tsallis, M. Gell-Mann, Y. Sato, Extensivity and entropy production, Europhysics News 36, 186 (2005) 186 (in: J.P. Boon, C. Tsallis (Eds.), Nonextensive Satistical Mechanics: New Trends, New Perspectives, Europhysics News (special issue), 2005.

9. T. Kodama, H.-T. Elze, C.E. Augiar and T. Koide, Europhys. Lett. 70, 439 (2005); T. Kodama, J. Phys. G 31, S1051 (2005).

10. T. Laštovička, Eur. Phys. J. C 24, 529 (2002).

11. G. Wilk and Z. Włodarczyk, Phys. Rev. Lett. 84, 2770 (2000).

12. G. Wilk and Z. Włodarczyk, Chaos, Solitons and Fractals 13/3, 581 (2001).

13. F.S. Navarra, O.V. Utyuzh, G. Wilk, and Z. Włodarczyk, Nuovo Cimento Soc. Ital. Fis., C 24, 725 (2001).

14. C. Beck, E.G.D. Cohen, Physica A 322, 267 (2003); F. Sattin, Eur. Phys. J. B 49, 219 (2006).

15. O.V. Utyuzh, G. Wilk and Z. Włodarczyk, J. Phys. G 26, L39 (2000).

16. G. Wilk and Z. Włodarczyk, Physica A 305, 227 (2002);

17. M. Rybczyński, Z. Włodarczyk and G. Wilk, Nucl. Phys. (Proc. Suppl.) B 122, 325 (2003);

18. T. Osada, O.V. Utyuzh, G. Wilk and Z. Włodarczyk, Europ. Phys. J. B 50, 7 (2006).

19. F.S. Navarra, O.V. Utyuzh, G. Wilk and Z. Włodarczyk, Phys. Rev. D 67, 114002 (2003).

20. F.S. Navarra, O.V. Utyuzh, G. Wilk and Z. Włodarczyk, Physica A 344, 568 (2004);

21. M. Biyajima, M. Kaneyama, T. Mizoguchi and G. Wilk, Eur. Phys. J. C 40, 243 (2005); M. Biyajima, T. Mizoguchi, N. Nakajima, N. Suzuki, and G. Wilk, Eur. Phys. J. C 48, 593 (2006).

22. G. Wilk and Z. Włodarczyk, Physica A 376, 279 (2007).

23. I. Bediaga, E.M. Curado and J.M.de Miranda, Physica A 286, 156 (2000).

24. W.M. Alberico, A. Lavagno and P. Quarati, Eur. Phys. J. C 12, 499 (2000);

25. T. Wibig and I. Kurp, J. High Energy Phys. 12, 039 (2003).

26. A. Lavagno, Physica A 305, 238 (2002); W. M. Alberico, P. Czerski, A. Lavagno, M. Nardi, and V. Somá, Physica A 387, 467 (2008).

27. C.E. Aguiar and T. Kodama, Physica A 320, 371 (2003). 28. B. De, S. Bhattacharyya, G. Sau and S.K. Biswas, Int. J. Mod. Phys. E 16 (2007) 1687.

29. T. Sherman and J. Rafelski, Lecture Notes in Physics 633, 377 (2004).

30. T.S. Biró and G. Purcsel, Phys. Rev. Lett. 95162302 (2005); Phys. Lett. A 372, 1174 (2008). See also: T.S. Biró, Abstract composition rule for relativistic kinetic energy in the thermodynamical limit, arXiv: 0809.4675 [nucl-th].

31. T.S. Biró and G. Kaniadakis, Eur. Phys. J. B 50, 3 (2006) and references therein.

32. C. Beck, Physica A 286, 164 (2000).

33. G. Wilk and Z. Włodarczyk, Nucl. Phys. B (Proc. Suppl.) 75A, 191 (1999).

34. G. Wilk and Z. Włodarczyk, Phys. Rev. D 50, 2318 (1994). 35. H. Heiselberg et al., Phys. Rev. Lett. 67, 2946 (1991); B. Blättel et al., Phys. Rev. D 47, 2761 (1993); L. Frankfurt, V. Guzey and M. Strikman, J. Phys. G 27, R23 (2001).

36. L. Frankfurt, M. Strikman, D. Treleani and C. Weiss, Evidence for color fluctuations in the nucleon in high-energy collisions, arXiv:0808.0182[hep-ph] (and in preparation). 
37. L.D. Landau and I.M. Lifschitz, Course of Theoretical Physics: Hydrodynamics, Pergamon Press, New York 1958 or Course of Theoretical Physics: Mechanics of Continous Media, Pergamon Press, Oxford 1981.

38. T.S. Biró and A. Jakovác, Phys. Rev. Lett. 94, 132302 (2005).

39. Y.-A. Chao, Nucl. Phys. B 40, 475 (1972); Y.M. Shabelski, R.M. Weiner, G. Wilk, and Z. Włodarczyk, J. Phys. G 18, 1281 (1992); F.O. Durães, F.S. Navarra and G. Wilk, Braz. J. Phys. 35, 3 (2005).

40. C. De Marzo et al., Phys. Rev. D 26, 1019 (1982) and D 29, 2476 (1984); R. Baltrusaitis et al.,Phys. Rev. Lett. 52,1380 (1993); F. Abe et al., Phys. Rev. D 41, 2330 (1990).

41. R. Barate, et al., (ALEPH Collab.), Phys. Rep. 294, 1 (1998).

42. S.V. Afanasjev et al. (NA49 Collab.), Phys. Rev. C 66, 054902 (2002).

43. B.B. Beck et al. (PHOBOS Coll.), Phys. Rev. Lett. 91, 052303 (2003).

44. T.T. Chou and C.N. Yang, Phys. Rev. Lett. 54, 510 (1985); Phys. Rev. D 32, 1692 (1985).

45. P. Carruthers and C.S. Shih, Int. J. Mod. Phys. A 2, 1447 (1986).

46. C. Albajar et al. (UA1 Collab.) Nucl. Phys. B 335, 261 (1990)

47. T. Alber et al (NA35 Collaboration), Eur. Phys. J. C 2, 643 (1998).

48. L.D. Landau and I.M. Lifschitz, Course of Theoretical Physics: Statistical Physics, Pergmon Press, New York 1958.

49. L. Stodolsky, Phys. Rev. Lett. 75, 1044 (1995); S. Mrówczyński, Phys. Lett. B 430, 9 (1998); E.V. Shuryak, Phys. Lett. B 423, 9 (1998).

50. M. Stephanov, K. Rajagopal and E. Shuryak, Phys. Rev. Lett. 81, 4816 (1998) and Phys. Rev. D 60, 114028 (1999); S. Mrówczyński, Phys. Rev. C 57, 1518 (1998).

51. T.C.P. Chui, D.R. Swanson, M.J. Adriaans, J.A. Nissen and J.A. Lipa, Phys. Rev. Lett. 69, 3005 (1992); C. Kittel, Physics Today 5, 93 (1988); B.B. Mandelbrot, Physics Today 42, 71 (1989); H.B. Prosper, Am. J. Phys. 61, 54 (1993); G.D.J. Phillies, Am. J. Phys. 52, 629 (1984).

52. K. Kadaja and M. Martinis, Z. Phys. C 56, 437 (1992).

53. R. Hagedorn, Nuovo Cim. Suppl. 3, 147 (1965); Nuovo Cim. A 52, 64 (1967) and CERN Report 71-12, 1971.

54. S.S. Adler et al. (PHENIX Coll.), Phys. Rev. C 69 (2004) 034909; J. Adams et al. (STAR Coll.) Phys. Lett. B 616, 8 (2005) and Phys.Lett. B 637, 161 (2006.

55. C. Alt et al. (NA49 Collaboration), Phys. Rev. C 75, 064904 (2007).

56. E. G. Ferreiro, F. del Moral, and C. Pajares, Phys. Rev. C 69, 034901 (2004).

57. M. Rybczyński and Z. Włodarczyk, J. Phys. Conf. Ser. 5, 238 (2005).

58. M. Gaździcki and M. Gorenstein, Phys. Lett. B 640, 155 (2006) (2006).

59. C. Tsallis, R.S. Mendes and A.R. Plastino, Physica A261, 534 (1998).

60. S. Abe, Phys. Lett. A 275, 250 (2000).

61. S. Abe, Physica A 368, 430 (2006).

62. W. Broniowski, B. Hiller, W. Florkowski, and P. Bożek, Phys. Lett. B 635, 290 (2006); F. Jinghua et al, Phys. Rev. C 72, 017901 (2005); J. Adams et al. (STAR Collab.), Phys. Rev. C 72, 044902 (2005); K. Adcox et al., (PHENIX Collab.), Phys. Rev. C 66, 024901 (2002).
63. M. Rybczyński et al. (NA49 Collab.), J. Phys. Conf. Ser. 5, 74 (2005)

64. G.Wilk and Z.Włodarczyk, AIP Conference Proceedings 965, $76(2007)$

65. M.P. Almeida, Physica A 300, 424 (2001) and 325, 426 (2003).

66. Fu Jinghua, Meng Ta-chung, R. Rittel and K. Tabelow, Phys. Rev. Lett. 86, 1961 (2001).

67. M. Rybczyński, Z. Włodarczyk and G. Wilk, Nucl. Phys. (Proc. Suppl.) B 97, 81 (2001).

68. G. Wilk and Z. Włodarczyk, Acta Phys. Polon. B 35, 871 (2004).

69. G. Wilk and Z. Włodarczyk, Acta Phys. Polon. B 35, 2141 (2004).

70. M. Gaździcki and M.I. Gorenstein, Phys. Lett. B 517, 250 (2001).

71. A. Białas, Phys. Lett. B 466, 301 (1999).

72. T. Osada and G. Wilk, Phys. Rev. C 77, 044903 and Prog. Theor. Phys. Suppl. 174, 168 (2008); see also Dissipative or just Nonextensive hydrodynamics? - Nonextensive/Dissipative correspondence -, arXiv:0805.2253 nuclphys] (to be published in Indian Journal of Physics). 